\title{
A novel streak velocimetry technique based on 2D fits of decaying phosphor particle images
}

\author{
Luming Fan 1,2,3, Patrizio Vena ${ }^{2}$, Bruno Savard ${ }^{3,4}$, Guangtao Xuan 1 , Benoît Fond ${ }^{1, *}$ \\ ${ }^{1}$ Otto-von-Guericke-Universität Magdeburg, Universitätsplatz 2, 39106, Germany \\ ${ }^{2}$ National Research Council (NRC), 1200 Montreal Road, Ottawa ON K1A 0R6, Canada \\ 3University of Ottawa, 75 Laurier Ave., Ottawa, ON K1N 6N5, Canada \\ 4Polytechnique Montréal, 2500 Chemin de Polytechnique, Montréal, QC H3T 1J4, Canada
}

\begin{abstract}
A new 2D velocimetry technique based on streaks formed by individual phosphor particles, which are moving during their luminescence decay following pulsed excitation is proposed in this study. Tin-doped phosphor particles $(\mathrm{Sr}, \mathrm{Mg})_{3}(\mathrm{PO} 4)_{2}: \mathrm{Sn}^{2+}$ are dispersed into flows and excited by a pulsed UV light sheet. During the phosphor decay time $(\sim 27 \mu \mathrm{s})$, the emission streaks due to particle motion are recorded. A 2D fitting is then applied on each particle streak against the analytical expression of intensity distribution, to obtain the velocity information for each particle. Unlike Particle Tracking Velocimetry (PTV) this technique does not rely on any particle image searching procedure.
\end{abstract}

Introduction. Near-wall velocity measurements still remain a great challenge to the velocimetry community today, because (a) laser reflection could easily damage the camera; (b) laser flare and reflection from the wall strongly deteriorates the vector detection probability; and (c) as a window-based technique, PIV cannot resolve the fine length scales as required by boundary layer studies. To solve these problems, we propose a new velocimetry technique based on individual phosphor particle streaks in the present study.

Particle streak velocimetry (PSV) is, however, not a new concept, but used as a very old expedient method before two-head pulsed lasers were available to researchers. In that method, particles were illuminated by a continuous light source and their trajectories were recorded on films by a camera with a fixed exposure time. The length of these streaks were then measured manually and converted to velocity vectors. This manual process is very onerous and time-consuming, and in particular, the accuracy cannot be guaranteed as the particle start and end position cannot be determined accurately due to light diffraction. Later on, similar PSV techniques based on fluorescent-dyed particles or quantum-dots were also introduced. However, soon after double pulse PIV lasers and double-frame CCD cameras were invented and commercialised, PSV was no longer popular, and instead PIV became the state-of-the-art tool for planar velocimetry.

However, 40 years since then, the computational capability of PCs and image processing tools have gone through tremendous progress. Therefore, we revisit the particle streak velocimetry technique based on phosphor particle tracers, and use a 2D fitting to extract the initial particle position and the two components of velocity with sub-pixel accuracy. For near-wall measurement, in particular, the incident laser wavelength is rejected by phosphorescence spectral filter, so the problem of laser flare and damage to the camera can be avoided. The proposed technique relies on 2D parametric fitting, by which the contribution from background signal can be recognised and does not affect the extraction of velocity components. Further, since the PSV technique is based on individual particle tracking, and has subpixel resolution $(<10 \mu \mathrm{m})$ on particle position determination, velocity information within the near-wall boundary layer can be well resolved.

In this study, we firstly introduce the theory of the phosphor streak velocimetry. Then we demonstrate the PSV technique in an air jet, and compare it with the PIV result, conducted simultaneously using a green PIV laser (Fig.1). Finally, we show some results obtained from a near-wall velocity measurement.

Methods. The PSV setup is shown in Figure 1. Particles of $(\mathrm{Sr}, \mathrm{Mg})_{3}\left(\mathrm{PO}_{4}\right)_{2}: \mathrm{Sn}^{2+}$ seeded into an air flow are imaged with a sCMOS camera at a magnification of 0.3 for a field of view of $2.7 \times 2.3 \mathrm{~cm}^{2}$. The decay time is around $27 \mu \mathrm{s}$ between $300-500 \mathrm{~K}$ [1], which provides a velocity dynamic range of $0.5-7 \mathrm{~m} / \mathrm{s}$. Nevertheless, the PSV technique can be easily extended to flows with higher/lower velocities by selecting a proper candidate that has a shorter/longer decay time from a long list of phosphor materials.

The intensity distribution of a still particle image is expressed by the Airy function, and is usually approximated as a 2D Gaussian function. Adding velocity components $v_{x}$ and $v_{y}$, time $t$ and a decay constant $\tau$ to this 2D Gaussian function reads:

$$
I(x, y, t)=I_{0} \cdot \exp (-t / \tau) \cdot \exp \left[-\frac{\left(x-x_{0}-v_{x} t\right)^{2}+\left(y-y_{0}-v_{y} t\right)^{2}}{2 \sigma^{2}}\right]
$$


Integrate this equation over $t$ from 0 to infinity yields a set function of 6 variables: $I_{0}$ is the intensity constant, $x_{0}, y_{0}$ are initial particle position, $\sigma$ is the particle image size constant. Those parameters are extracted for each recorded particle streak by least square fitting. Local background signals can be estimated from the intensity PDF in each streak window, or by applying a threshold to the intensity gradient.

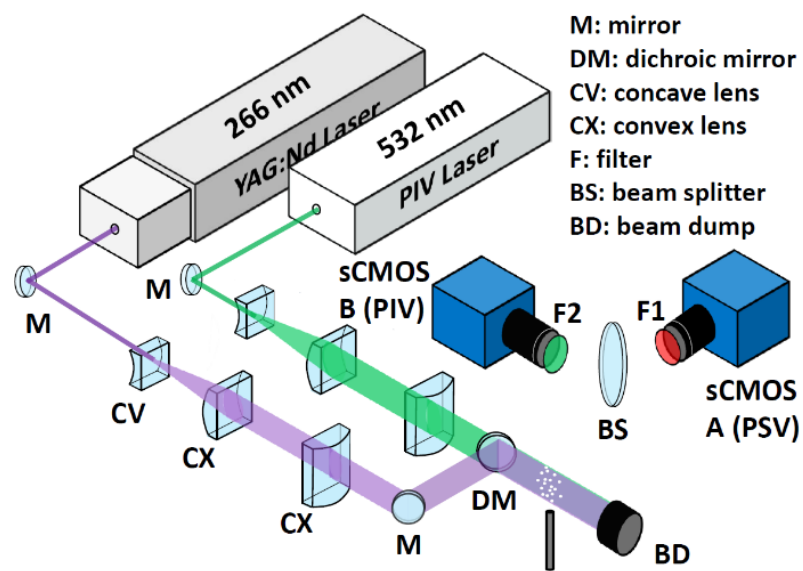

Figure 1 Schematic view of experimental setup
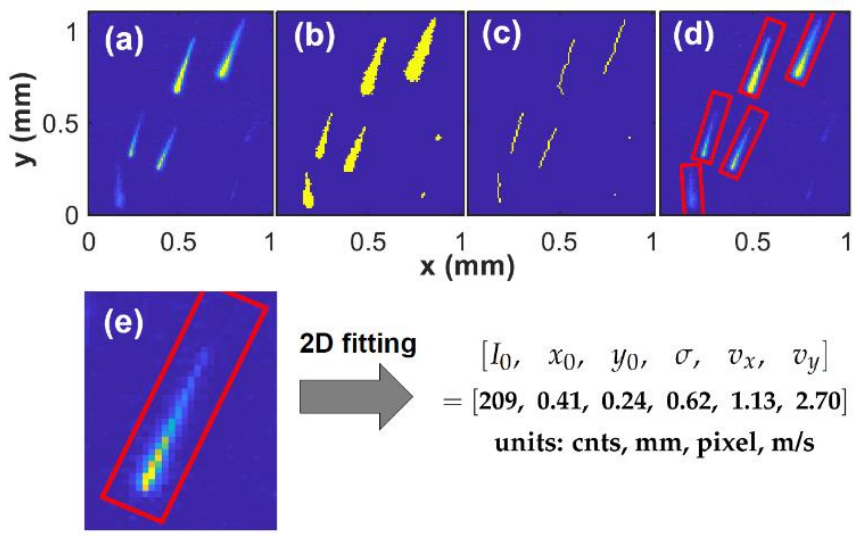

2D fitting $\quad\left[I_{0}, x_{0}, y_{0}, \quad \sigma, v_{x}, v_{y}\right]$ $=[209,0.41,0.24,0.62,1.13,2.70]$ units: cnts, $\mathrm{mm}$, pixel, $\mathrm{m} / \mathrm{s}$

Figure 2 The image processing procedure and an example streak fitting

Results. Figure 2 shows the image processing method and an example streak fitting. Figure 3 provides (a) an example single-shot, and (b) accumulated vector field in a $R e=1500$ open jet obtained by phosphor streak velocimetry. Figure 3(c) and (d) show the comparison between the PSV and PIV results at different flow velocities. Within $0.5-7 \mathrm{~m} / \mathrm{s}$ velocity range, the PSV results based on Tin-doped phosphor matched well with those obtained by PIV. Figure 4(a) presents the accumulated near-wall vector field measured by PSV, the closest vector measured is only $30 \mu \mathrm{m}$ from the wall, as shown in Figure 4(b). Figure 4(c) shows the comparison between the measured velocity profile across the boundary layer and the theoretical Blasius solution, which shows excellent agreement.
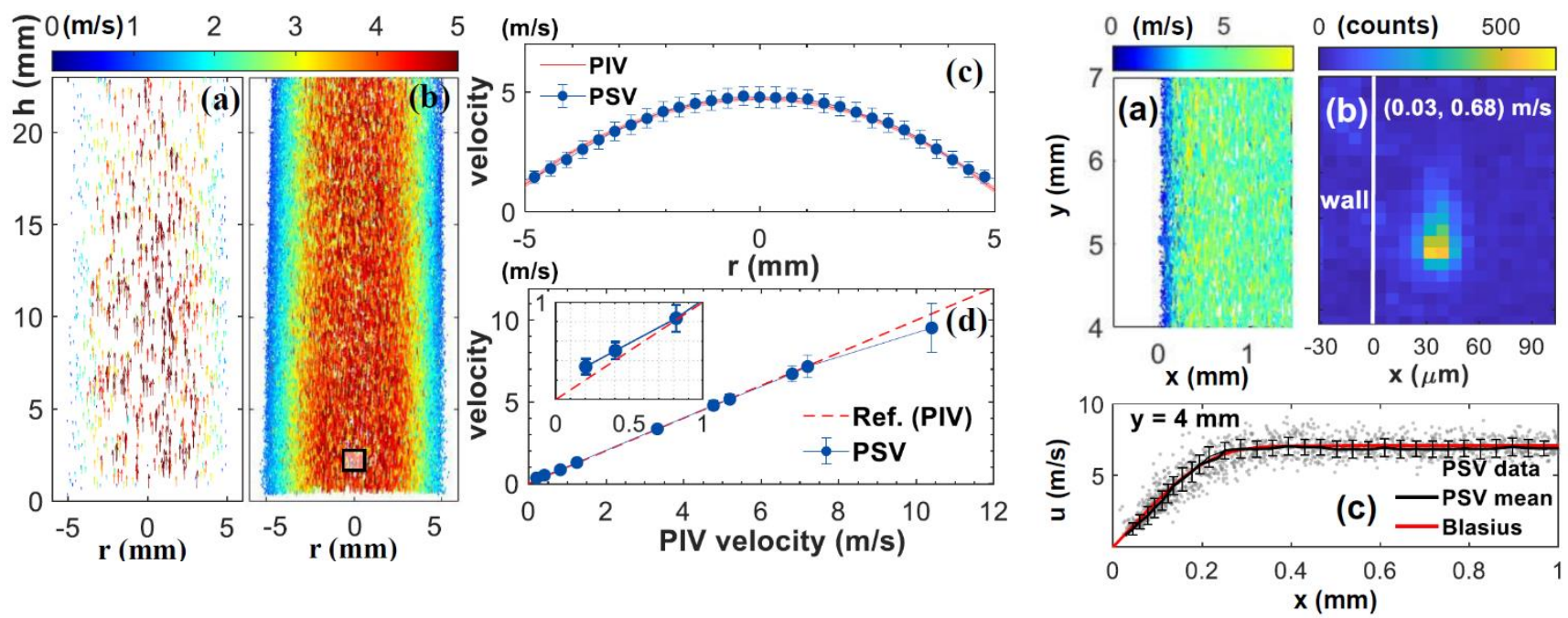

Figure 3 (a) Single shot, and (b) accumulated PSV vector field; (c) comparison of mean axial velocity profiles, and (d) comparison of PSV and PIV results in the square region at different flow velocities

Figure 4 Near-wall boundary layer measurement, and a comparison with the Blasius solution

Conclusions. In this study, a new velocimetry technique is proposed, based on phosphor particle streaks and 2D least-square fitting to the analytical expression of the emission trajectory. The technique is first demonstrated in an open jet where results were compared to PIV before being applied in a $200 \mu \mathrm{m}$ thick boundary layer. Experiments had very good agreement and showed that the new PSV technique can be applied to characterise the flow field very close to the wall. Further, considering the ratio-based thermometry that has been well established for phosphor particles, simultaneous temperature imaging can be implemented by adding another camera, which will be presented in future work.

\section{References}

[1] B.Fond, C.Abram, M, Pougin, $\mathrm{F}$, Beyrau. Investigation of the tin-doped phosphor $(\mathrm{Sr}, \mathrm{Mg})_{3}\left(\mathrm{PO}_{4}\right)_{2}: \mathrm{Sn}^{2+}$ for fluid temperature measurements. Opt. Mat. Express. 9 802-818 (2019). 